\title{
ON ONE-SIDED LIPSCHITZ STABILITY OF SET-VALUED CONTRACTIONS
}

\author{
S. Adly, ${ }^{1}$ A. L. Dontchev, ${ }^{2}$ and M. Théra ${ }^{1}$ \\ ${ }^{1}$ Laboratoire XLIM, Université de Limoges, Limoges Cedex, France \\ ${ }^{2}$ Mathematical Reviews, Ann Arbor, Michigan, USA
}

We give conditions under which the distance from a point $x$ to the set of fixed points of the composition of the set-valued mappings $F$ and $G$ is bounded by a constant times the smallest distance between $F^{-1}(x)$ and $G(x)$. This estimate allows us to significantly sharpen a result by T.-C. Lim [10] regarding fixed-points stability of set-valued contractions. A global version of the Lyusternik-Graves theorem is obtained from this estimate as well. We apply the generalization of Lim's result to establish one-sided Lipschitz properties of the solution mapping of a differential inclusion with a parameter.

Keywords Composition; Differential inclusions; Fixed points; Lyusternik-Graves theorem; Lipschitz stability; Set-valued mappings.

In a path-breaking paper, Teck-Cheong Lim [10] showed that the Pompeiu-Hausdorff distance between the sets of fixed points of two setvalued contraction mappings is bounded by a constant times the uniform Pompeiu-Hausdorff distance between the mappings. Specifically, he proved the following result:

Lim's Lemma (Lim [10, Lemma 1]). Let X be a complete metric space and let $T_{1}$ and $T_{2}$ map $X$ into the family of nonempty closed subsets of $X$. Suppose that both $T_{1}$ and $T_{2}$ are Lipschitz continuous on $X$ with the same Lipschitz constant 
$\lambda \in[0,1)$. Then

$$
\text { haus }\left(\operatorname{Fix}\left(T_{1}\right), \operatorname{Fix}\left(T_{2}\right)\right) \leq \frac{1}{1-\lambda} \sup _{x \in X} \operatorname{haus}\left(T_{1}(x), T_{2}(x)\right) .
$$

Before going further, let us fix the notation and terminology. Throughout this article, $X$ and $Y$ are metric spaces unless stated otherwise. Any metric is denoted by $\rho(\cdot, \cdot)$, any norm by $\|\cdot\|$, and $d(x, C)$ is the distance from a point $x \in X$ to a subset $C \subset X$; that is $d(x, C)=$ $\inf \left\{\rho\left(x, x^{\prime}\right) \mid x^{\prime} \in C\right\}$ whenever $C \neq \emptyset$ and $d(x, \emptyset)=\infty$. The closed ball centered at $x$ with radius $r$ is denoted by $\mathbb{B}_{r}(x)$, and the closed unit ball is $\mathbb{B}$. The excess from a set $C$ to a set $D$ is $e(C, D)=\sup _{x \in C} d(x, D)$ under the convention $e(\emptyset, D)=0$ for $D \neq \emptyset$ and $e(D, \emptyset)=+\infty$ for any $D$; thus, if $e(C, D)<\infty$ then $D$ must be nonempty. The Pompeiu-Hausdorff distance between $C$ and $D$ is haus $(C, D)=\max \{e(C, D), e(D, C)\}$. The smallest distance between two sets $C$ and $D$ is denoted by $\mathbf{d}(C, D)$, that is, $\mathbf{d}(C, D)=\inf \left\{\rho\left(x^{\prime}, x\right) \mid x^{\prime} \in C, x \in D\right\}$. If one of the sets $C$ and $D$ is empty, we set $\mathbf{d}(C, D)=$ haus $(C, D)=+\infty$. Given a set $D \subset X \times Y$ we denote by $P_{X}(D)$ the projection of $D$ on $X$, that is, $P_{X}(D)=\{x \in X \mid \exists y \in Y$ such that $(x, y) \in D\}$.

Any mapping, possibly set-valued and acting from a space $X$ to (the subsets of) a space $Y$, is denoted by $F: X \rightrightarrows Y$. The domain of $F$ is $\operatorname{dom} F=\{x \in X \mid F(x) \neq \emptyset\}$, its range is $\operatorname{rge} F=\{y \mid \exists x$ with $y \in F(x)\}$, and its graph is gph $F=\{(x, y) \in X \times Y \mid y \in F(x)\}$. The inverse $F^{-1}: Y \rightrightarrows$ $X$ of a mapping $F: X \rightrightarrows Y$ is defined as $F^{-1}(y)=\{x \mid y \in F(x)\}$. By a composition of a mappings $B: Z \rightrightarrows Y$ with a mapping $A: X \times P \rightrightarrows Z$ we mean a mapping $C=B \circ A: X \times P \rightrightarrows Y$ whose graph contains all points $(x, p, y) \in \operatorname{gph} C$ for which there exists $z \in Z$ such that $(x, p, z) \in \operatorname{gph} A$ and $(z, y) \in \operatorname{gph} B$; in other words, $C(x, p)=\{y \in B(z) \mid z \in A(x, p)\}$. We denote the set of fixed points of a mapping $F: X \rightrightarrows X$ by $\operatorname{Fix}(F)$; that is, $\operatorname{Fix}(F):=$ $\{x \in X \mid x \in F(x)\}$. Note that for mappings $F: Y \rightrightarrows X$ and $G: X \rightrightarrows Y$ we have $\operatorname{Fix}(F \circ G)=\left\{x \in X: F^{-1}(x) \cap G(x) \neq \emptyset\right\}$.

A set-valued mapping $F: X \rightrightarrows Y$ is said to be Lipschitz continuous relative to a set $D \subset \operatorname{dom} F$ when $F$ is closed valued ${ }^{1}$ on $D$ and there exists a constant $\kappa \geq 0$ (Lipschitz constant) such that

$$
\text { haus }\left(F(x), F\left(x^{\prime}\right)\right) \leq \kappa \rho\left(x, x^{\prime}\right) \quad \text { for all } x, x^{\prime} \in D \text {. }
$$

When $D=X$ we say that $F$ is Lipschitz continuous. In that case, $\operatorname{dom} F=$ $X$. If a mapping $F$ acting from a metric space $X$ to a complete metric space

${ }^{1}$ Following [7], we require that Lipschitz continuous mappings must be closed valued to avoid the situation when Lipschitz continuity does not entail continuity. This is also assumed implicitly in $[10]$. 
$Y$ is Lipschitz continuous, then $F$ necessarily has a closed graph. Indeed, let $\left(x_{k}, y_{k}\right) \in \operatorname{gph} F$ and $\left(x_{k}, y_{k}\right) \rightarrow(x, y)$; then

$$
\begin{aligned}
d(y, F(x)) \leq & \rho\left(y, y_{k}\right)+d\left(y_{k}, F\left(x_{k}\right)\right)+\text { haus }\left(F\left(x_{k}\right), F(x)\right) \leq \rho\left(y, y_{k}\right) \\
& +\kappa \rho\left(x_{k}, x\right) \rightarrow 0,
\end{aligned}
$$

and, hence, $(x, y) \in \operatorname{gph} F$.

It is well documented in the literature that the Lipschitz continuity of a mapping $F$ is equivalent to two properties of its inverse $F^{-1}$ : metric regularity and linear openness. Without going into this further, we use the fact established in [7, Proposition 3C.1] that a mapping $G: Y \rightrightarrows X$ is Lipschitz continuous with constant $\kappa$ if and only if

$$
d(x, G(y)) \leq \kappa d\left(y, G^{-1}(x)\right) \quad \text { for every } x \in X, \quad y \in Y .
$$

Going back to Lim's lemma [10] we started with, first observe that it implies the standard Nadler fixed point theorem for set-valued mappings [11]; indeed, if $T_{1}=T_{2}=T$ then the right side of (1) is zero and, hence, so is the left side, thus $\operatorname{Fix}(T)$ is nonempty. Right after the statement of his lemma, Lim stated a theorem (Theorem 1) which follows immediately from the lemma and which Lim apparently considered as the central result in his paper. This theorem says that when $\left\{T_{n}\right\}$ is a sequence of set-valued mappings $T_{n}: X \rightrightarrows X$ each of which is Lipschitz continuous with the same Lipschitz constant $\lambda \in[0,1)$, then

$$
\lim _{n \rightarrow \infty} \sup _{x \in X} \text { haus }\left(T_{n}(x), T_{0}(x)\right)=0 \Longrightarrow \lim _{n \rightarrow \infty} \operatorname{haus}\left(\operatorname{Fix}\left(T_{n}\right), \operatorname{Fix}\left(T_{0}\right)\right)=0 .
$$

In Theorems 2 and 3 in [10], Lim presented extensions of this implication in some particular spaces and compact-valued nonexpansive mappings. Finally, he applied his Theorem 1 to show continuity of the solution mapping for a differential inclusion with respect to the initial conditions.

Observe that Lim's lemma is a quantitative result and, therefore, it is a natural tool to establish quantitative stability of perturbed fixed points. Specifically, the following corollary of Lim's lemma shows Lipschitz continuity of fixed points:

Theorem 2 (Lipschitz continuity of fixed points). Let $X$ be a complete metric space and $P$ be a metric space. Consider a mapping $M: P \times X \rightrightarrows X$ with the following properties:

(i) $M(p, \cdot)$ is Lipschitz continuous with a Lipschitz constant $\lambda \in[0,1)$ uniformly in $p \in P$; 
(ii) $M(\cdot, x)$ is Lipschitz continuous with a Lipschitz constant $\mu$ uniformly in $x \in$ $X$.

Then the mapping $p \mapsto \operatorname{Fix}(M(p, \cdot))$ is Lipschitz continuous with a constant $\mu /(1-\lambda)$.

Proof. We will first show that for each $p \in P$ the set $\operatorname{Fix}(M(p, \cdot))$ is closed. Let $u_{k} \in \operatorname{Fix}(M(p, \cdot))$ for all $k$ and $u_{k} \rightarrow u$. Then $u_{k} \in M\left(p, u_{k}\right)$ for all $k$. We have

$$
\begin{aligned}
d(u, M(p, u)) & \leq d\left(u, M\left(p, u_{k}\right)\right)+\operatorname{haus}\left(M\left(p, u_{k}\right), M(p, u)\right) \\
& \leq \rho\left(u, u_{k}\right)+\lambda \rho\left(u, u_{k}\right) \rightarrow 0 \text { as } k \rightarrow \infty .
\end{aligned}
$$

Hence, $d(u, M(p, u))=0$, that is, $u \in M(p, u)$, which implies that $u \in$ $\operatorname{Fix}(M(p, \cdot))$, thus $\operatorname{Fix}(M(p, \cdot))$ is closed.

Let $p_{1}, p_{2} \in P$. Applying Lim's lemma with $T_{1}(x)=M\left(p_{1}, x\right)$ and $T_{2}(x)=M\left(p_{2}, x\right)$ we have

$$
\begin{aligned}
\operatorname{haus}\left(\operatorname{Fix}\left(M\left(p_{1}, \cdot\right)\right), \operatorname{Fix}\left(M\left(p_{2}, \cdot\right)\right)\right) & \leq \frac{1}{1-\lambda} \sup _{x \in X} \operatorname{haus}\left(M\left(p_{1}, x\right), M\left(p_{2}, x\right)\right) \\
& \leq \frac{\mu}{1-\lambda} \rho\left(p_{1}, p_{2}\right) .
\end{aligned}
$$

As a corollary of our main result presented in Theorem 6, we will obtain the following generalization of Lim's lemma, where the PompeiuHausdorff distance is replaced by either of the excesses.

Theorem 3 (generalized Lim's lemma). On the conditions of Lim's lemma,

$$
e\left(\operatorname{Fix}\left(T_{i}\right), \operatorname{Fix}\left(T_{j}\right)\right) \leq \frac{1}{1-\lambda} \sup _{x \in X} e\left(T_{i}(x), T_{j}(x)\right) \quad \text { for } i, j=1,2 .
$$

Theorem 3 allows us to obtain results that are parallel to Theorem 2 but for the weaker one-sided Lipschitz continuity property. First, we need the following definition:

Definition. A mapping $F: X \rightrightarrows Y$ is said to be outer [resp. inner] Lipschitz continuous at $\bar{x}$ relative to a set $D \subset \operatorname{dom} F$ when $\bar{x} \in D$ and there exists a constant $\mu \geq 0$ such that

$$
e(F(x), F(\bar{x})) \leq \mu \rho(x, \bar{x}) \quad[\operatorname{resp} . e(F(\bar{x}), F(x)) \leq \mu \rho(x, \bar{x})] \quad \text { for all } x \in D .
$$

When $D=X$ they we say that $F$ is outer [resp. inner] Lipschitz continuous at $\bar{x}$. In that case, $\operatorname{dom} F=X$. 
Observe that both the outer and the inner Lipschitz continuity are much weaker properties than the Lipschitz continuity. Directly from the definition, when $F$ is both outer and inner Lipschitz continuous at a point, then it is Lipschitz continuous. The property of outer Lipschitz continuity was introduced by Robinson [12] under the name "upper Lipschitz continuity," the main motivation being the important result due to him that every mapping $F: \mathbb{R}^{n} \rightrightarrows \mathbb{R}^{m}$ whose graph is the union of finitely many polyhedral convex sets is outer Lipschitz continuous at any point of its domain.

From Theorem 3 we can obtain the following result, which strengthens Theorem 2.

Theorem 4. Let $X$ be a complete metric space and $P$ be a metric space. Consider a mapping $M: P \times X \rightrightarrows X$ with the following properties:

(i) $M(p, \cdot)$ is Lipschitz continuous with a Lipschitz constant $\lambda \in[0,1)$ uniformly in $p \in P$;

(ii) $M(\cdot, x)$ is outer [resp. inner] Lipschitz continuous at $\bar{p}$ with a constant $\mu$ uniformly in $x \in X$.

Then the mapping $p \mapsto \operatorname{Fix}(M(p, \cdot))$ is outer [resp. inner] Lipschitz continuous at $\bar{p}$ with a constant $\mu /(1-\lambda)$.

Proof. Applying Theorem 3 with $T_{1}(x)=M(p, x)$ and $T_{2}(x)=M(\bar{p}, x)$ we have

$e\left(\operatorname{Fix}\left(M(p, \cdot), \operatorname{Fix}(M(\bar{p}, \cdot)) \leq \frac{1}{1-\lambda} \sup _{x \in X} e(M(p, x), M(\bar{p}, x)) \leq \frac{\mu}{1-\lambda} \rho(p, \bar{p})\right.\right.$.

Arutyunov derived in [1] a coincidence theorem which we present here in the following form, where the assumptions about completeness/closedness of graphs are different from those originally used:

Theorem 5 (Arutyunov [1, Theorem 2]). Consider mappings $F: Y \rightrightarrows X$ and $G: X \rightrightarrows Y$ and assume that one of the set $\operatorname{gph} F$, gph $G$ is complete and the other is closed. Let $\kappa$ and $\mu$ be nonnegative constants such that $\kappa \mu<1$. Suppose that $F$ is Lipschitz continuous with constant $\kappa$ and $G$ is Lipschitz continuous with constant $\mu$. Then for every $\varepsilon>0$ and every $x \in X$ there exists a point $\xi \in X$ such that

$$
F^{-1}(\xi) \cap G(\xi) \neq \emptyset \quad \text { and } \quad \rho(x, \xi) \leq \frac{\kappa}{1-\kappa \mu} \mathbf{d}\left(F^{-1}(x), G(x)\right)+\varepsilon .
$$


The main part of the result given next as Theorem 6; namely, the implication (i) $\Rightarrow$ (ii), is equivalent, up to some adjustments regarding closedness/completeness of sets, to Arutyunov's theorem.

Theorem 6 (estimate for fixed points of composition). Let $\kappa$ and $\mu$ be nonnegative constants such that $\kappa \mu<1$. Consider a mapping $F: Y \rightrightarrows X$ with closed and nonempty graph and $P_{X}(\operatorname{gph} F)$ complete. Then the following are equivalent:

(i) The mapping $F$ is Lipschitz continuous with constant $\kappa$;

(ii) $\operatorname{dom} F=Y$ and for any mapping $G: X \rightrightarrows Y$ with closed graph which is Lipschitz continuous with constant $\mu$ and such that $P_{Y}(\mathrm{gph} G)$ is complete, the following inequality holds:

$$
d(x, \operatorname{Fix}(F \circ G)) \leq \frac{\kappa}{1-\kappa \mu} \mathbf{d}\left(F^{-1}(x), G(x)\right) \quad \text { for every } x \in X .
$$

Proof. Let (i) hold, let $G$ be as required in (ii) and let $x \in X$. If $x \notin \operatorname{rge} F$ or $G(x)=\emptyset$, the right side of (6) is $+\infty$ and we are done. Otherwise, let $y \in G(x)$. Choose $\varepsilon>0$. Since by definition $\operatorname{dom} F=Y$, there exists $u \in$ $F(y)$ such that $\rho(x, u)<d(x, F(y))+\varepsilon$. If $u=x$ then $x \in \operatorname{Fix}(F \circ G)$ and the left side of (6) is zero, so there is nothing more to prove. Otherwise, we have

$$
d(x, F(y))<(1+\varepsilon) \rho(u, x) .
$$

Denote $a:=(1+\varepsilon) \rho(u, x)$; then

$$
a \leq(1+\varepsilon)(d(x, F(y))+\varepsilon) .
$$

We will construct a sequence $\left\{\left(x^{k}, y^{k}\right)\right\}$ with the following properties:

$$
y^{k} \in F^{-1}\left(x^{k+1}\right) \cap G\left(x^{k}\right)
$$

and

$$
\rho\left(y^{k}, y^{k-1}\right)<\mu a(\kappa \mu)^{k-1}, \quad \rho\left(x^{k+1}, x^{k}\right)<a(\kappa \mu)^{k} .
$$

Let $x^{0}=x$ and $y^{0}=y$. In the first step, observe that, from (7),

$$
d\left(x^{0}, F\left(y^{0}\right)\right)<a .
$$

Then there exists $x^{1} \in F\left(y^{0}\right)$ with

$$
\rho\left(x^{1}, x^{0}\right)<a(\kappa \mu)^{0} .
$$


Furthermore, since

$$
d\left(y^{0}, G\left(x^{1}\right)\right) \leq \operatorname{haus}\left(G\left(x^{0}\right), G\left(x^{1}\right)\right) \leq \mu \rho\left(x^{0}, x^{1}\right)<\mu a,
$$

there exists $y^{1} \in G\left(x^{1}\right)$ such that

$$
\rho\left(y^{0}, y^{1}\right)<\mu a(\kappa \mu)^{0} .
$$

From the Lipschitz continuity of $F$, we have

$$
d\left(x^{1}, F\left(y^{1}\right)\right) \leq \text { haus }\left(\left(y^{0}\right), F\left(y^{1}\right)\right) \leq \kappa \rho\left(y^{0}, y^{1}\right)<\kappa \mu a(\kappa \mu)^{0}=a(\kappa \mu) .
$$

Hence, there exists $x^{2} \in F\left(y^{1}\right)$ with

$$
\rho\left(x^{2}, x^{1}\right)<a(\kappa \mu)^{1} .
$$

We obtain (9) and (10) for $k=1$.

Proceeding by induction, suppose that we have already found a sequence $\left\{\left(x^{k}, y^{k}\right)\right\}$ satisfying (9) and (10) for $k=1, \ldots, j$, for some $j>1$. Then

$$
d\left(y^{j}, G\left(x^{j+1}\right)\right) \leq \operatorname{haus}\left(G\left(x^{j}\right), G\left(x^{j+1}\right)\right) \leq \mu \rho\left(x^{j}, x^{j+1}\right)<\mu a(\kappa \mu)^{j} .
$$

Hence, one can find $y^{j+1} \in G\left(x^{j+1}\right)$ such that

$$
\rho\left(y^{j}, y^{j+1}\right)<\mu a(\kappa \mu)^{j}
$$

The Lipschitz continuity of $F$ gives us

$d\left(x^{j+1}, F\left(y^{j+1}\right)\right) \leq$ haus $\left(F\left(y^{j}\right), F\left(y^{j+1}\right)\right) \leq \kappa \rho\left(y^{j}, y^{j+1}\right)<\kappa \mu a(\kappa \mu)^{j}=a(\kappa \mu)^{j+1}$.

Then there exists $x^{j+2} \in F\left(y^{j+1}\right)$ with

$$
\rho\left(x^{j+2}, x^{j+1}\right)<a(\kappa \mu)^{j+1} .
$$

The induction step is complete.

For $k>m+1>1$, from (10) we have

$$
\rho\left(x^{k}, x^{m}\right) \leq \sum_{i=m}^{k-1} \rho\left(x^{i+1}, x^{i}\right)<\frac{a(\kappa \mu)^{m}}{1-\kappa \mu}
$$

and

$$
\rho\left(y^{k}, y^{m}\right) \leq \sum_{i=m}^{k-1} \rho\left(y^{i+1}, y^{i}\right)<\frac{\mu a(\kappa \mu)^{m}}{1-\kappa \mu} .
$$


Hence, $\left\{\left(x^{k}, y^{k}\right)\right\}$ is a Cauchy sequence. Since $\left(x^{k}, y^{k}\right)$ belongs to the complete set $P_{X}(\operatorname{gph} F) \times P_{Y}(\operatorname{gph} G)$ for all $k$ we conclude that this sequence is convergent to some $(\hat{x}, \hat{y})$. Since both $F$ and $G$ have closed graphs, we have $\hat{x} \in F(\hat{y})$ and $\hat{y} \in G(\hat{x})$, hence $\hat{x} \in \operatorname{Fix}(F \circ G)$.

Note that

$$
\rho\left(x^{k}, x\right) \leq \sum_{i=0}^{k-1} \rho\left(x^{i+1}, x^{i}\right)<a \sum_{i=0}^{k-1}(\kappa \mu)^{i}<\frac{a}{(1-\kappa \mu)} .
$$

Passing to the limit with $k \rightarrow \infty$, from (8) we obtain

$$
d(x, \operatorname{Fix}(F \circ G)) \leq \rho(\hat{x}, x) \leq \frac{(1+\varepsilon)(d(x, F(y))+\varepsilon)}{1-\kappa \mu} .
$$

Since $\varepsilon$ can be arbitrarily small, we get

$$
d(x, \operatorname{Fix}(F \circ G)) \leq \frac{1}{1-\kappa \mu} d(x, F(y)) .
$$

This combined with (3) gives us

$$
d(x, \operatorname{Fix}(F \circ G)) \leq \frac{\kappa}{1-\kappa \mu} d\left(y, F^{-1}(x)\right) .
$$

Taking into account that $y$ can be arbitrarily chosen in $G(x)$ we obtain (6).

Now, suppose that (ii) is satisfied. Let $y, y^{\prime} \in Y$ and let $x \in F(y)$. Choose $G(x)=y^{\prime}$ for all $x \in X$. Clearly, $P_{Y}(\operatorname{gph} G)$ is complete and $G$ is Lipschitz continuous with constant $\mu=0$; moreover, $d(x, \operatorname{Fix}(F \circ G))=d\left(x, F\left(y^{\prime}\right)\right)$. From (6),

$$
d\left(x, F\left(y^{\prime}\right)\right) \leq \kappa d\left(y^{\prime}, F^{-1}(x)\right) \leq \kappa \rho\left(y, y^{\prime}\right) .
$$

Taking supremum with respect to $x \in F(y)$ in the left side of this inequality, we obtain that $F$ is Lipschitz continuous.

Remark 1. Note that the estimate (6) is sharp in the sense that if the left side is zero, so is the right side.

Remark 2. One of the referees of the first version of this paper suggested the following proof that (a part of) Theorem 6 follows from Theorem 5. Put $U=P_{X}(\operatorname{gph} F)$ and apply Theorem 5 with $F$ being the identity mapping from $U$ to $U$ and $G$ identified with the composition $F \circ G$ restricted on $U$, which is Lipschitz continuous with Lipschitz constant $\kappa \mu<$ 1 . For any $x \in U$ from Theorem 5 we have

$$
d(x, \operatorname{Fix}(F \circ G)) \leq \frac{1}{1-\kappa \mu} d(x,(F \circ G)(x)) .
$$


Further, for any $y \in G(x)$ we have

$$
d(x, \operatorname{Fix}(F \circ G)) \leq d(x, F(y)) .
$$

Combining the last two estimates with (3) and noting that $y$ is an arbitrary point in $G(x)$, we obtain (5). When $x \notin U$ the right side of (5) is $+\infty$ and there is nothing more to prove.

Remark 3. Lim proved his lemma in [10] by using an iterative procedure which is quite different from the one used in the proof of Theorem 6 . In particular, this procedure involves a construction of just one sequence of iterates $x^{k}$ for both mappings $T_{1}$ and $T_{2}$. Also, the iteration we use is related but different from the one in [1].

Proof of Theorem 3. Apply Theorem 6 with $X=Y, F=I$, the identity mapping, and $G=T_{1}$. Then $G$ is Lipschitz continuous with Lipschitz constant $\mu \in[0,1)$ and from (6) we have that for any $x \in X$,

$$
d\left(x, \operatorname{Fix}\left(T_{1}\right)\right) \leq \frac{1}{1-\mu} d\left(x, T_{1}(x)\right) .
$$

Let $T_{2}: X \rightrightarrows Y$ be Lipschitz continuous with Lipschitz constant $\mu$. Clearly, (12) holds for every $x \in \operatorname{Fix}\left(T_{2}\right)$ and, hence, taking supremum with respect to such $x$, we get

$$
e\left(\operatorname{Fix}\left(T_{2}\right), \operatorname{Fix}\left(T_{1}\right)\right) \leq \frac{1}{1-\mu} \sup _{x \in T_{2}(x)} d\left(x, T_{1}(x)\right) \leq \sup _{x \in X} \frac{1}{1-\mu} e\left(T_{2}(x), T_{1}(x)\right) .
$$

By symmetry, the proof is complete.

Proof of Theorem 5. It is easy to modify the proof of Theorem 6 for the case when one of the sets gph $F$, gph $G$ is complete while the other is closed. Indeed, if gph $F$ is complete, then $\left(x^{k}, y^{k}\right)$ is convergent, and hence the limit $(\hat{x}, \hat{y})$ satisfies $\hat{x} \in F(\hat{y})$ and $\hat{y} \in G(\hat{x})$, Let $x \in X$ and $\varepsilon>0$. From (6), there exists $\xi \in(F \circ G)(\xi)$, or equivalently, $F^{-1}(\xi) \cap G(\xi) \neq \emptyset$, such that

$$
\rho(x, \xi) \leq \frac{\kappa}{1-\kappa \mu} \mathbf{d}\left(F^{-1}(x), G(x)\right)+\varepsilon .
$$

Another application of Theorem 6 is the following result which is a version of a general paradigm in nonlinear analysis the most popular form of which appears in the basic inverse function theorem, for more see [7, chap. 5]. A local version of it stems from early works of Lyusternik and Graves and is most known as the Lyusternik-Graves theorem. Here we 
deduce from Theorem 6 the following global version of the LyusternikGraves theorem:

Theorem 7 (Global Lyusternik-Graves). Let $X$ be a complete metric space and $Y$ be a Banach space. Consider mappings $\Phi: X \rightrightarrows Y$ and $\Psi: X \rightrightarrows Y$ and suppose that both $\Phi^{-1}$ and $\Psi$ are globally Lipschitz continuous with constant $\kappa>0$ and $\mu>0$, respectively. If $\kappa \mu<1$ then

$$
d\left(x,(\Phi+\Psi)^{-1}(y)\right) \leq \frac{\kappa}{1-\kappa \mu} d(y,(\Phi+\Psi)(x)) \quad \text { for all }(x, y) \in X \times Y .
$$

Proof. Choose $y \in Y$ and let $u \in \operatorname{Fix}\left(\Phi^{-1} \circ(-\Psi+y)\right)$; then there exists $z \in-\Psi(u)+y$ with $u \in \Phi^{-1}(z)$. Hence, $y \in \Psi(u)+z \subset \Psi(u)+\Phi(u)$, that is, $u \in(\Phi+\Psi)^{-1}(y)$. Thus, $\operatorname{Fix}\left(\Phi^{-1} \circ(-\Psi+y)\right) \subset(\Phi+\Psi)^{-1}(y)$. Therefore, for any $x \in X$ we have

$$
d\left(x,(\Phi+\Psi)^{-1}(y)\right) \leq d\left(x, \operatorname{Fix}\left(\Phi^{-1} \circ(-\Psi+y)\right)\right)
$$

Applying Theorem 6 with $F=\Phi^{-1}$ and $G(\cdot)=-\Psi(\cdot)+y$ to the last inequality, from (6) we get

$$
\begin{aligned}
d\left(x, \operatorname{Fix}\left(\Phi^{-1} \circ(-\Psi+y)\right)\right) & \leq \frac{\kappa}{1-\kappa \mu} \mathbf{d}(\Phi(x),-\Psi(x)+y) \\
& \leq \frac{\kappa}{1-\kappa \mu} d(y, \Phi(x)+\Psi(x)) .
\end{aligned}
$$

There is a vast literature on fixed points of compositions which, however, cover topics that are different from the subject of the present paper. Results related to the ones obtained in the current paper are given in $[2-5,8,9,13,14]$.

In his paper, Lim [10, Theorem 4] presented an application of his Theorem 1 given by (4) to obtain a stability result for the solution mapping of an initial value problem for a differential inclusion. Specifically, he proved continuity of the set of solutions, in the space of the continuous functions equipped with the supremum norm, with respect to the initial condition. However, Lim did not mention anything about Lipschitz continuity of the solution set although such a statement easily follows from his analysis. Also, some of his assumptions he maid are not necessary to obtain such a result. In further lines we will first present the setting of Lim's result (Theorem 4) with some adjustments in terminology and notation, and then show how to obtain Lipschitz continuity. Then we generalize this result to one-sided Lipschitz stability of the solution mapping with respect to a parameter. In order to simplify the presentation, we work in the Euclidean space $\mathbb{R}^{n}$ and consider an autonomous differential inclusion over a finite time interval. 
Let $a>0$ and let $B$ be a bounded set in $\mathbb{R}^{n}$. Consider a mapping $R$ acting from $B$ into the family of nonempty closed subsets of $B$ which is Lipschitz continuous with respect to the Pompeiu-Hausdorff metric with a Lipschitz constant $\kappa$; that is,

$$
\text { haus }(R(x), R(y)) \leq \kappa\|x-y\| \quad \text { for all } x, y \in \mathbb{B} \text {. }
$$

Given an initial point $b \in B$, consider the initial-value problem

$$
\dot{x}(t) \in R(x(t)) \quad \text { for a.e. } t \in[0, a], \quad x(0)=b .
$$

By a solution of (14), we mean any absolutely continuous function which satisfies (14) for almost every (a.e.) $t \in[0, a]$, with $x(0)=b$. Let $C([0, a], B)$ be the space of continuous functions on the interval $[0, a]$ with values in $B$, equipped with the usual supremum norm $\|\cdot\|_{C}$. Define the mapping

$$
\begin{aligned}
& C([0, a], B) \ni x \mapsto T(b, x)=\{y \in C([0, a], B) \mid \text { there exists a Lebesgue } \\
& \text { measurable function } z \text { such that } z(\tau) \in R(x(\tau)) \text { a.e. } \tau \in[0, a] \\
& \text { such that } \left.y(t)=b+\int_{0}^{t} z(\tau) d \tau \text { for all } t \in[0, a]\right\}
\end{aligned}
$$

where the integral is in the sense of Lebesgue.

We will first show that $T(b, x) \neq \emptyset$ when $x \in C([0, a], B)$. Choose any $x \in C([0, a], B)$. For each $t \in[0, a]$, let $z(t)$ be a projection of $0 \in \mathbb{R}^{n}$ on the closed set $R(x(t))$. Since both $x$ and $R$ are continuous, we obtain that the function $z$ defined in such a way is measurable; since it is uniformly bounded, it is integrable. Then $y \in T(b, x)$ when $y(t)=b+{ }_{0}^{t} z(\tau) d \tau$ for $t \in[0, a]$.

To prove Lipschitz continuity of $T(b, \cdot)$ we use a similar argument. Let $x_{1}, x_{2} \in C([0, a], B)$ and let $y_{1} \in T\left(b, x_{1}\right)$. From the very definition of the mapping $T$, there exists a measurable selection $z_{1}$ of $R\left(x_{1}\right)$ such that $y_{1}(t)=b+{ }_{0}^{t} z_{1}(\tau) d \tau$ for all $t \in[0, a]$. For each $t \in[0, a]$, let $z_{2}(t)$ be a projection of $z_{1}(t)$ on $R\left(x_{2}(t)\right)$. Since $z_{1}$ is measurable and $R$ is continuous, we obtain that the function $z_{2}$ defined in such a way is measurable; since it is uniformly bounded, it is integrable. Let $y_{2}(t)=b+\int_{0}^{t} z_{2}(\tau) d \tau$ for $t \in$ $[0, a]$. Then, from the Lipschitz continuity property (13) of $R$ we obtain

$$
\left\|y_{1}-y_{2}\right\|_{C} \leq \int_{0}^{a}\left\|z_{1}(\tau)-z_{2}(\tau)\right\| d \tau \leq \kappa \int_{0}^{a}\left\|x_{1}(\tau)-x_{2}(\tau)\right\| d \tau \leq \kappa a\left\|x_{1}-x_{2}\right\|_{C} .
$$


Hence, the mapping $T(b, \cdot)$ is Lipschitz continuous with a Lipschitz constant $\kappa a$ which is independent of $b$. Let $b_{1}, b_{2} \in B$ and denote $T_{1}(x)=$ $T\left(b_{1}, x\right)$ and $T_{2}(x)=T\left(b_{2}, x\right)$. Observe that

$$
\text { haus }\left(T_{1}(x), T_{2}(x)\right) \leq\left\|b_{1}-b_{2}\right\| \text {. }
$$

Indeed, let $y_{1} \in T_{1}(x)$. Then there exists an associate selection $z$ of $R(x)$ such $y_{1}(t)=b_{1}+\int_{0}^{t} z(\tau) d \tau$ for all $t \in[0, a]$. Clearly $y_{2}$ defined as $y_{2}(t)=$ $b_{2}+\int_{0}^{t} z(\tau) d \tau, t \in[0, a]$, is from $T_{2}(x)$. This yields $(15)$.

Denote by $S(b)$ the set of solutions of the differential inclusion (14) for a fixed initial condition $b \in B$. Then $S(b)=\operatorname{Fix}(T(b, \cdot))$. From Lim's lemma we obtain the following theorem:

Theorem 8. Consider the differential inclusion (14) for a Lipschitz continuous mapping $R$ with a Lipschitz constant $\kappa$ as in (13). Choose $a>0$ such that $\kappa a<1$. Then the solution mapping $S$ of (14) is Lipschitz continuous from the set $B$ to the collection of nonempty subsets of functions from the space $C([0, a], B)$.

In Lim's paper [10], it is assumed that $R$ is in addition convex-valued and the conclusion is that the solution mapping $S$ is merely continuous, obtained as an application of [10, Theorem 1].

We can obtain even more general results for a differential inclusion with a parameter, that is,

$$
\dot{x}(t) \in R(p, x(t)) \quad \text { for a.e. } t \in[0, a], \quad x(0)=b,
$$

where now $b \in B$ is fixed and $p$ is a parameter from a metric space $P$ with a reference value $\bar{p}$. By repeating the above argument where we use Theorem 4 instead of Lim's lemma, we obtain:

Theorem 9. Consider the differential inclusion (16), where the mapping $R$ is Lipschitz continuous with respect to $x \in B$ with constant $\kappa$, uniformly in $p \in P$, and outer [resp. inner] Lipschitz continuous with respect to $p$ at $\bar{p}$ with constant $\mu$, uniformly in $x \in B$. Let $\kappa a<1$. Then the solution mapping

$$
P \ni p \mapsto S(p)=\{x \in C([0, a], B) \mid x \text { solves }(16) \text { for } p \in P\}
$$

is outer [resp. inner] Lipschitz continuous at $\bar{p}$ with a constant $\mu /(1-\kappa a)$.

Theorem 9 can be easily extended to a nonautonomous setting, as in Lim's original paper [10], or even in a much more general context of infinite-dimensional spaces. Specifically, in a series of papers A. Cernea considered various nonlinear differential inclusions and proved stability of 
their solutions by using Lim's lemma. In particular, in [6] he considered nonlinear integrodifferential inclusions of the following form:

$$
\dot{x}(t) \in F(t, x(t), V(x)(t)) \quad \text { for a.e. } t \in[0, \infty), \quad x(0)=x_{0},
$$

where $F:[0,+\infty) \times X \times X \rightrightarrows X, X$ is a separable Banach space and $V: C([0,+\infty), X) \rightarrow C([0,+\infty), X)$ is the nonlinear Volterra integral operator

$$
V(x)(t)=\int_{0}^{t} k(s, x(s)) d s,
$$

where $k:[0,+\infty) \times X \rightarrow X$ is Lipschitz continuous. Applying Lim's lemma, Cernea proved Lipschitz continuity of the Lipschitz mapping on an infinite time horizon. To do that, instead of considering sufficiently small time interval as in Theorem 8 above, he used a weighted $L^{2}$ space with a sufficiently large weight to obtain Lipschitz continuity of the mapping defining the inclusion (17) with a Lipschitz constant less than 1. Then he followed the approach based on Lim's lemma. By using the extension of Lim's lemma in this article, this result can be extended to differential inclusion of the form (17) involving a parameter $p \in P$ and employing, as in Theorem 9, the outer [resp. inner] Lipschitz continuity.

\section{ACKNOWLEDGMENTS}

The authors wish to thank the anonymous referees for their valuable comments and suggestions.

\section{FUNDING}

A. L. Dontchev was supported by the National Science Foundation, Grant DMS 1008341 through the University of Michigan. M. Théra was Supported by Grant DP 11010211 of the Australian Research Council through the University of Ballard.

\section{REFERENCES}

1. A. V. Arutyunov (2007). Covering mappings in metric spaces and fixed points. Dokl. Akad. Nauk 416:151-155 [Russian]. English translation: Doklady Math. 76:665-668.

2. A. V. Arutyunov, E. Avakov, B. Gelman, A. Dmitruk, and V. Obukhovskii (2009). Locally covering maps in metric spaces and coincidence points. Journal of Fixed Point Theory and Applications 5:105-127.

3. A. V. Arutyunov (2009). Stability of coincidence points and properties of covering mappings. Mat. Zametki 86:153-158 [Russian]. English translation: Math. Notes 86:153-158.

4. A. V. Arutyunov (2009). Stability of coincidence points and set-valued covering maps in metric spaces. Dokl. Acad. Nauk 80:555-557 [Russian]. English translation: Doklady Math. 80:555-557. 
5. D. Azé and J.-P. Penot (2006). On the dependence of fixed point sets of pseudo-contractive multifunctions. Application to differential inclusions. Nonlinear Dyn. Syst. Theory 6:31-47.

6. A. Cernea (2008). Stability of solution sets of nonlinear integrodifferential inclusions. Rev. Roumaine Math. Pures Appl. 53:277-283.

7. A. L. Dontchev and R. T. Rockafellar (2009). Implicit Functions and Solution Mappings. Springer, Dordrecht.

8. M. Durea and R. Strugariu (2012). Openness stability and implicit multifunction theorems: Applications to variational systems. Nonlinear Anal. 75:1246-1259.

9. A. D. Ioffe (2010). Towards variational analysis in metric spaces: Metric regularity and fixed points. Math. Program. B 123:241-252.

10. T.-C. Lim (1985). On fixed-point stability for set-valued contractive mappings with applications to generalized differential equations. J. Math. Anal. Appl 110:436-441.

11. S. B. Nadler, Jr. (1969). Multi-valued contraction mappings. Pacific J. Math. 30:475-488.

12. S. M. Robinson (1979). Generalized equations and their solutions. I. Basic theory. Point-to-set maps and mathematical programming. Math. Programming Study 10:128-141.

13. S. L. Singh, S. N. Mishra, and W. Sinkala (2012). A note on fixed point stability for generalized set-valued contractions. Appl. Math. Letters 25:1708-1710.

14. Z. X. Yin and N. K. Fu (2009). Metric regularity of composite multifunctions in Banach spaces. Taiwanese J. Math. 13:1723-1735. 